\title{
HEMORRAGIA CEREBRAL NO TRAUMÁTICA EN ADULTO COMO PRIMERA MANIFESTACIÓN DEHEMOFILIA. REPORTE DECASO, HOSPITAL TEODORO MALDONADO CARBO
} NON-TRAUMATIC BRAIN HEMORRHAGEIN ADULTS AS THE FIRST MANIFESTATION OF HEMOPHILIA. CASE REPORT, TEODORO MALDONADO CARBO SPECIALTY HOSPITAL

HEMORRAGIA CEREBRAL NÃO TRAUMÁTICA EM ADULTOS COMO A PRIMEIRA MANIFESTAÇÃO DA HEMOFILIA. RELATO DE CASO, HOSPITAL ESPECIALIZADO DO TEODORO MALDONADO CARBO

\author{
LITO CAMPOS CARBO, KARLA WELLINGTON CRIOLLO, YAZMÍN SÁNCHEZ CEVALLOS, ALICIA MOLINA PANTOJA
}

Hospital de Especialidades Teodoro Maldonado Carbo; Guayaquil, Ecuador

\section{Resumen}

La hemorragia intracraneal en pacientes con hemofilia tiene una tasa de letalidad de aproximadamente $25 \%$, y en hasta un $40 \%$ de los pacientes afectados no registran antecedentes desencadenantes de sangrado tales como hipertensión arterial o traumatismos. Se reporta el caso clínico de un paciente atendido en el área de emergencia del hospital de especialidades Teodoro Maldonado Carbo en la ciudad de Guayaquil. Se trata de un hombre de 39 años, con antecedente de hipertensión arterial, que ingresa al area de emergencia con deterioro del nivel de conciencia; la tomografia computarizada de cerebro evidencia hemorragia intraparenquimatosa; se establece una sospecha inicialmente de etiología hipertesiva, sin embargo la prolongación del tiempo parcial de tromboplastina (TPT) canaliza el despistaje de trastornos de coagulación, obteniéndose entre los resultados deficiencia leve de factor VIII. La hemorragia intracraneal es de fácil diagnóstico gracias a la disponibilidad de la tomografía axial computarizada (TAC) y se relaciona a trauma en ams del $50 \%$ de los casos por lo que su asociación como complicación de hemofilia puede pasar desapercibida.

PALABRAS CLAVE: hemorragia intracraneal, trastornos de la coagulación, hemofilia, factor VIII.

Abstract

Intracranial hemorrhage in patients with hemophilia has a case fatality rate of $25 \%$, and $40 \%$ have no history of triggering bleeding such as high blood pressure or trauma. We report the clinical case of a patient treated in the emergency area of the Teodoro Maldonado Carbo specialty hospital in Guayaquil, a 39-year-old man with a history of high blood pressure who enters the emergency area with deterioration of conscience. Brain Computed Tomography shows intraparenchymal hemorrhage, an initial suspicion of hypertensive etiology is established, however the prolongation of the Part Time Thromboplastin (TPT) channels the screening of coagulation disorders. Obtaining among the results mild deficiency of coagulation factor VIII, in which restitution therapy is established with clotting factor VIII concentrates. Intracranial hemorrhage is easy to diagnose thanks to the availability of Computed Tomography (CT) and is related to trauma in more than $50 \%$ of cases, so its association as a complication of Hemophilia may go unnoticed. KEYWORDS: intracranial hemorrhage, blood coagulation disorders, hemophilia, factor VIII.

Resumo

Hemorragia intracraniana em pacientes com hemofilia tem uma taxa de letalidade de 25\%, e 40\% não têm histórico de sangramento, como pressão alta ou trauma. Relatamos o caso de um paciente tratado na área de emergência hospital de especialidades Teodoro Maldonado Carbo na cidade de Guayaquil, é um paciente de 39 anos com antecedente de hipertensão chega na área de emergência com deterioração do nível de consciência, tomografia computadorizada do cérebro mostra hemorragia intraparenquimatosa, uma suspeita inicial de etiologia hipertensiva é estabelecida, porém o prolongamento do Tempo de Tromboplastina Parcial (TPP) canaliza a triagem para distúrbios da coagulação. Os resultados mostram uma ligeira deficiência do fator VIII de coagulação, no qual a terapia de restituição com concentrados de fator VIII de coagulação é estabelecida. A hemorragia intracraniana é fácil de diagnosticar graças à disponibilidade da tomografia axial computadorizada (TC) e está relacionada ao trauma em mais de 50\% dos casos, portanto sua associação como complicação da hemofilia pode passar despercebida.

PALABRAS-CHAVE: hemorragia intracraniana, transtornos coagulação, hemofilia, fator VIII. 


\section{INTRODUCCIÓN}

La hemofilia es una enfermedad hereditaria caracterizada por deficiencia de factores de coagulación, la pricipal manifestación clínica es la hemorragia, cuyo grado depende del nivel del factor VIII o IX presente en el plasma. Por la intensidad del sangrado y el nivel del factor, la hemofilia se clasifica en tres tipos: hemofilia severa ( $<1 \%$ de actividad del factor), hemofilia moderada (de 1 a $5 \%$ de actividad del factor), hemofilia leve ( $>5 \%$ de actividad del factor). Los individuos con hemofilia severa por lo general experimentan hemorragias repetitivas y espontáneas. ${ }^{1}$

La presentación clínica tanto asintomática o con hemorragias severas incluyen aquellas afectaciones del sistema nervioso central (SNC). El diagnóstico oportuno así como la infusión de concentrados del factor de coagulación deficiente es de suma importancia para el control de la hemorragia. La deficiencia de factor VIII se conoce como hemofilia A o hemofilia clásica mientras que la deficiencia del factor IX es denominada hemofilia B o Enfermedad de Christmas. Las hemofilias se caracterizan por una tendencia hemorrágica directamente proporcional a la deficiencia del factor de coagulación implicado; así, el nivel funcional del factor deficiente permite clasificar la enfermedad en: grave $(<1 \%$ de la actividad), moderada (entre 1-5\%) y leve (entre $5-40 \%)^{2}$

La hemorragia del SNC puede ser la primera manifestación clínica en recién nacidos y niños mayores considerándose la principal causa de muerte en pacientes hemofílicos y la complicación menos frecuente. Se estima una incidencia entre $2.21 .3 \%$ asociados mayoritariamente a hemofilia A hasta en un $78 \%$ de los casos. ${ }^{3}$ La hemorragia espontánea del SNC es poco común excepto en los casos en los que existe antecedente reciente de hemorragia o una lesión anatómica relacionada que predispone a una hemorragia aguda. Para las personas con hemofilia leve, el riesgo de hemorragia intracraneal es por lo menos $50 \%$ que el que corren las personas con hemofilia severa. ${ }^{4}$

\section{CASO CLÍNICO}

Paciente masculino de 39 años de edad, antecedentes patológicos personales de hipertensión arterial, que es trasladado al servicio de emergencias del hospital Teodoro Maldonado Carbo en la ciudad de Guayaquil donde ingresa por presentar cuadro clínico de aproximadamente tres horas de evolución caracterizado por deterioro súbito del nivel de conciencia precedido de cefalea holocraneana sin antecedente de trauma.

El examen neurológico inicial evidencia escala de Glasgow 13/15, hemiparesia izquierda braquial $1 / 5$, crural $3 / 5$, pupilas isocóricas reactivas, lesión central del séptimo par izquierdo Bragman IV, lesión de sexto par craneal izquierdo, tono trofismo conservado, sensibilidad conservada.

Estudios complementarios de laboratorio reportan TPT 3, hemoglobina $14 \mathrm{~g} / \mathrm{dl}$, plaquetas 336000 .

Tomografía compuatarizada simple de cerebro revela imagen en relación a hematoma intraparenquimatoso a nivel de núcleos de la base del lado derecho, (Figura 1) y colapso ipsilateral del ventrículo lateral sin desviación de la línea media (Figura 2).

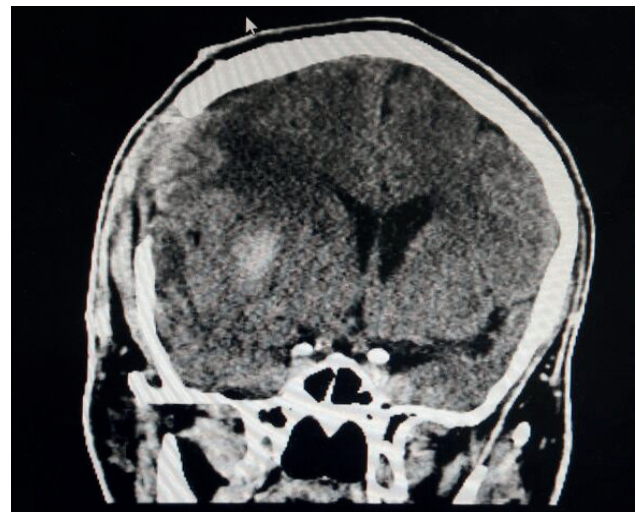

Figura 1. Hematoma intraparenquimatoso a nivel de núcleos de la base del lado derecho.

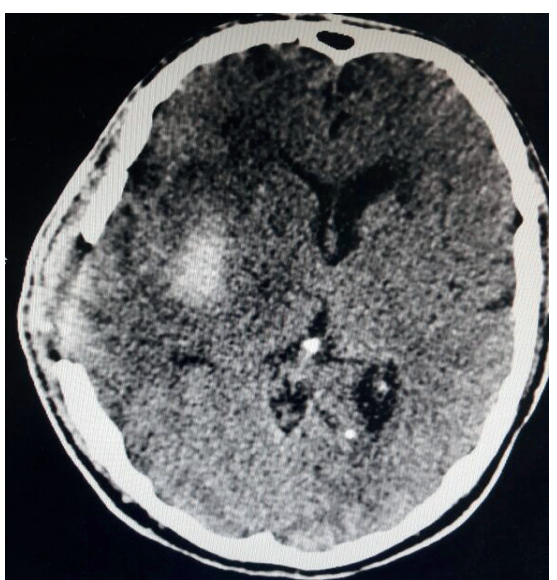

Figura 2. Hematoma intraparenquimatoso derecho. Colapso ventricular ipsilateral. $\quad \overline{\mathbf{1 3 9}}$ 
Se solicita dosaje factor VIII por prolongación de tiempo parcial de tromboplastina obteniéndose como resultado $27 \% \mathrm{UB}$.

Con diagnóstico de hemofilia A leve se inicia terapia de restitución con concentrado plas mático de factor VIII con el objetivo de elevar el nivel de factor a $100 \%$ por 7 días acorde a las guías para el tratamiento de la hemofilia de la Federación Mundial de Hemofilia, administrándose dosis de factor VIII 3500 U cada 12 horas.

Valorado por neurocirugía quien indica craniectomía descompresiva, la misma que se realiza sin complicaciones, con control óptimo de sangrado intraoperatorio, y requerimiento de soporte transfusional con 1 unidad de glóbulos rojos concentrados.

Se mantiene nivel de factor al $100 \%$ durante 7 días, luego se disminuye dosis para mantener nivel de factor al 50\% durante 21 días; durante este período no se presentó resangrado ni mayor deterioro neurológico; los datos de laboratorio reportaron tiempo parcial de tromboplastina en rangos normales.

En manejo multidisciplinario con el servicio de Neurología se inicia con profilaxis secundaria por seis meses con dosis de concentrado plasmático de factor VIII de 2500UI tres veces por semana y tratamiento con fisiatría por hemiparesia izquierda secuelar braquial 3/5, crural 4/5.

\section{DISCUSIÓN}

Este caso nos orienta a evaluar adecuadamente al paciente que cursa con manifestaciones hemorrágicas intracraneales en la búsqueda de la etiología del evento. La hemorragia en el SNC se presenta en el $8 \%$ de los pacientes hemofílicos, es una complicación poco común en pacientes adultos con una presentación espontánea del $2 \%$. Las localizaciones más frecuentes son intraparenquimatosa 37 a 46\%, subdural 27 a $44 \%$, subaracnoidea 5 a $16 \%$, intraventricular 10\%, epidural 3 a $11 \%$, de éstas las asociadas a peor pronóstico funcional son la intraparenquimatosa e intraventricular. ${ }^{5,6}$

En el presente reporte existe secuela funcional moderada sin registro secuelar cognitivo gracias a la instauración oportuna de la terapia sustitutiva de concentrados de factor VII ya que el inmediato inicio de tratamiento aumenta las posibilidades de un mejor pronóstico. ${ }^{6,7} \mathrm{La}$ terapia sustitutiva ha demostrado la capacidad de controlar esta complicación grave de la hemofilia siempre que se inicie rápidamente y se mantengan niveles elevados de factor VIII extendiendo su uso por periodos más largos en estos pacientes con hemorragia intracraneal. ${ }^{7}$

La tasa de mortalidad reportada de hemorragia intracraneal disminuye de $70 \%$ antes de 1960 a $20-30 \%$ en los 15 años siguientes y posiblemente ha disminuido aún más en las dos últimas décadas. Los estudios recientes indican que aunque la mortalidad ha disminuido se le atribuye la secuela neurológica en más del $75 \%$ de los casos y que afortunadamente el índice de recurrencia es poco común, junto el virus de la inmunodeficiencia humana (VIH) y la hepatitis $\mathrm{C}(\mathrm{VHC})$ contribuye en gran medida al acortamiento de la esperanza de vida de hemofílicos, manteniéndose como una causa sustancial de morbilidad en estos pacientes. ${ }^{8,9}$

Considerando los estudios realizados disponibles en la literatura y dado nuestro escenario la importancia de incluir en el diferencial de la etiología de la hemorragia cerebral la patología hemofílica en pacientes sin antecedentes conocidos de enfermedades hematológicas cuando exista alteración de los tiempos de coagulación o contaje de plaquetas debido a que para el inicio oportuno de la terapia sustitutiva debe realizarse el diagnóstico inicial adecuado.

El diagnóstico de hemofilia es fundamentalmente clínico ${ }^{2,7}$ y puede confirmarse con los exámenes de laboratorio de escrutinio y confirmatorios como el dosaje de factores de coagulación; ${ }^{10}$ no sólo los pacientes con hemofilia severa están afectados por hemorragia intracraneal; estudios presentados por The French Group demuestran que un tercio de los episodios se referían a pacientes con deficiencia leve en contraste con la experiencia brasileña en la cual 31 de 33 pacientes padecían hemofilia severa; esta diferencia podría obedecer a un sesgo originado por pacientes con hemofilia leve no diagnosticados. ${ }^{10,11}$

En referencia a la etiología de la hemorragia intracraneal en hemofílicos se acredita a traumatismo el $58.3 \%$ de los casos y como eventos espontáneos el 41.6\% ${ }^{12,13}$ Los médicos deben 
prestar especial atención al posible desarrollo de inhibidores después de tratamiento intensivo en pacientes afectados y demás estudios necesarios para evaluar la respuesta a la administración de los concentrados de factores de coagulación para determinar si el nivel plasmático de factor es el deseado. ${ }^{14,15}$

\section{CONCLUSIONES}

El diagnóstico rápido de hemorragia intracraneal en pacientes hemofílicos es crucial para su tratamiento y pronóstico, la búsqueda de factores contribuyentes, tanto los específicos de la hemofilia como los que favorecen el evento hemorrágico en la población general, es esencial para optimizar la atención terapéutica.El aspecto más importante en el manejo de la hemorragia intracraneal en pacientes hemofílicos es la terapia de reemplazo temprano sumado a la conducción multidisciplinaria.

Este tratamiento rápido aumentará las posibilidades de un mejor pronóstico. Otra medida de impacto consiste en la implementación de profilaxis secundaria con la administración del factor de coagulación deficiente, incluso cuando el episodio hemorrágico se considera menor, ya que esto evitaría la aparición de nuevas hemorragias.

\section{REFERENCIAS BIBLIOGRÁFICAS}

1. Amin, C., Sharathkumar, A., \& Griest, A. A. (2014). Bleeding diathesis and hemophilias. En E. Jose Biller and Jose M. Ferro, Handbook of Clinical Neurology. Neurologic Aspects of Systemic Disease Part II (págs. 1045-1059). Elsevier B.V.

2. García-Chávez, J., \& Majluf-Cruz, A. (2013). Hemofilia. Gaceta Médica de México, 308-321.

3. Hoots, W. K. (2007). Atención de Emergencias en casos de hemofilia. En F. M. Hemofilia, Tratamiento de la Hemofilia (págs. 1-16). Canadá: World Federation of Hemophilia.
4. M, P. T., Tagle M., P., Morales G., A., Pereira G., J., \& Huete L., I. (1996). Hematoma Intracerebral como priemra manifestación de Hemofilia. Revista Chilena de Pedaitría, 224-227.

5. S. Sagnier, B. T. (2015). Intracranial hemorrhage in tree haemophilics adults: A severe complication of hemophilia. Revue Neurologique.

6. de Tezanos Pinto M1, F. J. (1992). Update of 156 episodes of central nervous system bleeding in hemophiliacs. Haemostasis., 259-267.

7. Hemofilia, F. M. (2012). www.wfh.org. Obtenido de Guías para el tratamiento de la hemofilia: http:// www1.wfh.org/publications/files/pdf-1513.pdf

8. Bentancor N, L. E. (1992). Intracranial hemorrhage in hemophiliacs. Study of 10 episodes. Blood, 43-46.

9. Chorba TL, H. R. (1994). Changes in longevity and causes of death among persons with hemophilia A. American Journal of Hematology, 112-121.

10. N. Stieltjes, T. C. (2005). Intracranial haemorrhages in French haemophilia patients. Haemophilia, 452458.

11. Consenso de Médicos especialistas en hemofilia de la República Argentina. Guía de tratamiento de la Hemofilia. Primera Edición. Buenos Aires, Argentina; 2011. Disponible en: http://www.hemofilia.org. ar/archivos/pdfs/GuiaTrataᄀmientoHemofilia.pdf (último acceso 09 septiembre 2016).

12. Antunes SV1, V. P. (2003). Intracranial haemorrhage among a population of haemophilic patients in Brazil. Haemophilia., 573-577.

13. Jung Yoon Cho, W. S. (2016). Clinical Characteristics and Prognostic Factors in Hemophiliacs. Indian Journal of Hematology and Blood Transfusion.

14. M. Bladen, E. M. (2016). The incidence, risk and functional outcomes of intracranial haemorrhage in children with inherited bleeding disorders at one haemophilia center. Haemophilia, 1-8.

15. Char Witmer, R. P. (2010). Associations between intracranial haemorrhage and prescribed prophylaxis in a large cohort of haemophilia patients in the United States. British Journal of Hematology, 211-216. 